\title{
The Quest for Histology Driven Neoadjuvant Chemotherapy in Soft-Tissue Sarcoma: End of Road or Just the Beginning?
}

The role of chemotherapy in high-risk nonmetastatic soft-tissue sarcoma (STS) is modest as per the existing evidence. By and large, the chemotherapy regimen used in adjuvant and neoadjuvant setting had been a combination of anthracycline and ifosfamide. Increasingly simulating the approval of trabectedin and eribulin in specific histologies of STS, there is an ardent desire by sarcoma medical oncologists to sieve out the apt chemotherapy regimens for specific subtypes of sarcoma in adjuvant/neoadjuvant setting. ${ }^{[1,2]}$

In the current trial, Alessandro Gronchi et al. assessed histology tailored approach by randomly assigning 287 patients with localized high-risk extremity and trunk STS to receive 3 cycles of histology tailored chemotherapy in experimental arm as compared to control arm with ifosfamide and epirubicin. ${ }^{[3]}$ The histologies included in the trial were high-grade myxoid liposarcoma, leiomyosarcoma, synovial sarcoma, malignant peripheral nerve sheath tumors, and undifferentiated pleomorphic sarcoma given the background they represent $80 \%$ of all extremities and truncal STS and carry high metastatic risk. At the third futility analysis, after a short median follow-up of 12.3 months, the projected disease-free survival at 46 months was $62 \% \quad(95 \%$ confidence interval $[\mathrm{CI}]$ 48-77) in the standard chemotherapy group and 38\% (22-55) in the histotype-tailored chemotherapy group (stratified log-rank $P=0.004$; hazard ratio 2.00, 95\% CI $1 \cdot 22-3.26 ; P=0.006)$ and the trial was closed prematurely as superiority of histology tailored treatment was not expected. We congratulate the author for their endeavor for conducting histology-specific trial given the fact it is first trial in adjuvant and neoadjuvant setting with limited histologies. There are few pertinent points that we will like to highlight and should be kept in the mind while designing such trials in future and interpreting the result for day-to-day practice.

First, the histologies in this trial have distinct distribution as compared to the previous trials conducted by the same group. ${ }^{[3,4]}$ The percentage of synovial sarcoma patients was almost 3 times that of previous trial while the leiomyosarcoma patients were marginally more than previous trial. Although both histologies were in inclusion criteria, this finding is intriguing. This might make the comparability with previous trials with neoadjuvant chemotherapy less reliable and highlights the uniquely heterogeneous nature of STS in various trials.

Second, though authors acknowledge that the histotype-tailored regimens of etoposide plus ifosfamide and gemcitabine plus docetaxel were based on weaker data than the other three regimens we believe that the data even for other three regimens is not strong enough to conduct a phase 3 trial and has not been implemented adequately. Regarding trabectedin dose in myxoid liposarcoma arm, the dose is lower than Phase 2 trial which was conducted in upfront setting $\left(1.3 \mathrm{mg} / \mathrm{m}^{2}\right.$ in the current trial vs. $1.5 \mathrm{mg} / \mathrm{m}^{2}$ in Phase 2 study). In addition, the number of cycles used in Phase 2 trial was 4-6 and the best response achieved was beyond 3 cycles in two (of three patients who achieved complete remission). ${ }^{[5]}$ As late responses are also known to happen with trabectedin, may be three cycles are not sufficient for trabectedin. Similarly, gemcitabine and dacarbazine combination has been used in Phase 2 randomized trial as compared in dacarbazine in previously treated STS. ${ }^{[6]}$ This comes down to two distinct points first that this combination was not used in adriamycin naïve patients and its activity in leiomyosarcoma was just based on subgroup analysis. Thus, using gemcitabine and dacarbazine in a randomized trial in the first line seems unjust and certainly not evidence based. Similarly, although high-dose ifosfamide is active in synovial sarcoma, there is no study that has compared it with doxorubicin and ifosfamide combination prospectively in synovial sarcomas. We think before using in phase 3 trial, these questions should have been addressed in other phase 2 studies.

Although this trial was based upon the premises that three cycles of neoadjuvant chemotherapy are noninferior to five cycles, we must remember that trial in which it was proven had backbone of ifosfamide and epirubicin and not proven for other chemotherapy regimens and may be adjuvant chemotherapy would have helped in other chemotherapy regimens. ${ }^{[4]}$

Response rates in this study are also worth mentioning which is modest $16 \%$ and $11 \%$ in both arms. Previously in localized disease, the European Organisation for Research and Treatment of Cancer (EORTC) trial has reported 29\% response rates. Furthermore, in that EORTC trial ifosfamide was used in suboptimal doses and there was no preoperative radiation given unlike current trial. Although this could be attributed to heterogeneity, it is still worth mentioning that this trial had more synovial sarcoma patients.

In nutshell though this trial is one of the trials representing coming of age from one size fits all and could be harbinger of further such studies focused on histology but should be interpreted cautiously. Besides, it must not be taken as the evidence that neoadjuvant chemotherapy has shown to improve survival as that could be due to relative because of inferior survival in the experimental arm and being since only one-sided alpha was taken for sample size it was not 
designed to answer this question. In the end, further trials are required before we can implement any such strategy in practice.

\section{Aditi Aggarwal, Atul Sharma1, Sameer Rastogi ${ }^{1}$}

Department of Radiation Oncology, Lok Nayak Hospital and Maulana Azad Medical College, 'Department of Medical Oncology, IRCHH, AIIMS, New Delhi, India

Address for correspondence:

Dr. Sameer Rastogi, Department of Medical Oncology, IRCHH, AIIMS, New Delhi, India. E-mail:samdoc_mamc@yahoo.com

\section{References}

1. Press Announcements - FDA Approves First Drug to Show Survival Benefit in Liposarcoma. Available from: https://www. fda.gov/newsevents/newsroom/pressannouncements/ucm 483714 . htm. [Last accessed on 2017 Sep 20].

2. Press Announcements - FDA Approves New Therapy for Certain Types of Advanced Soft Tissue Sarcoma. Available from: https:// www.fda.gov/newsevents/newsroom/pressannouncements/ ucm468832.htm. [Last accessed on 2017 Sep 20].

3. Gronchi A, Ferrari S, Quagliuolo V, Broto JM, Pousa AL, Grignani $\mathrm{G}$, et al. Histotype-tailored neoadjuvant chemotherapy versus standard chemotherapy in patients with high-risk soft-tissue sarcomas (ISG-STS 1001): An international, open-label, randomised, controlled, phase 3, multicentre trial. Lancet Oncol 2017;18:812-22.
4. Gronchi A, Bui BN, Bonvalot S, Pilotti S, Ferrari S, Hohenberger $\mathrm{P}$, et al. Phase II clinical trial of neoadjuvant trabectedin in patients with advanced localized myxoid liposarcoma. Ann Oncol 2012;23:771-6.

5. Ducoulombier A, Cousin S, Kotecki N, Penel N. Gemcitabine-based chemotherapy in sarcomas: A systematic review of published trials. Crit Rev Oncol Hematol 2016;98:73-80.

6. Gronchi A, Frustaci S, Mercuri M, Martin J, Lopez-Pousa A, Verderio $\mathrm{P}$, et al. Short, full-dose adjuvant chemotherapy in high-risk adult soft tissue sarcomas: A randomized clinical trial from the Italian sarcoma group and the Spanish sarcoma group. J Clin Oncol 2012;30:850-6.

This is an open access journal, and articles are distributed under the terms of the Creative Commons Attribution-NonCommercial-ShareAlike 4.0 License, which allows others to remix, tweak, and build upon the work non-commercially, as long as appropriate credit is given and the new creations are licensed under the identical terms.

\begin{tabular}{|l|l|}
\hline \multicolumn{2}{|c|}{ Access this article online } \\
\hline Quick Response Code: & Website: \\
& www.ijmpo.org \\
\cline { 2 - 2 } & DOI: \\
\hline
\end{tabular}

How to cite this article: Aggarwal A, Sharma A, Rastogi S. The quest for histology driven neoadjuvant chemotherapy in soft-tissue sarcoma: End of road or just the beginning?. Indian J Med Paediatr Oncol 2018;39:427-8. 